General Mathematics Vol. 28, No. 1 (2020), 85-103

DOI: $10.2478 / \mathrm{gm}-2020-0007$

Sciendo

\title{
Some applications of generalized Ruscheweyh derivatives involving a general fractional derivative operator to a class of analytic functions with negative coefficients II ${ }^{1}$
}

\author{
Waggas Galib Atshan, S. R. Kulkarni
}

\begin{abstract}
In this paper, we study a class of univalent functions $f$ as defined by making use of the generalized Ruscheweyh derivatives involving a general fractional derivative operator, satisfying

$$
\operatorname{Re}\left\{\frac{z\left(\mathbf{J}_{1}^{\lambda, \mu} f(z)\right)^{\prime}}{(1-\gamma) \mathbf{J}_{1}^{\lambda, \mu} f(z)+\gamma z^{2}\left(\mathbf{J}_{1}^{\lambda, \mu} f(z)\right)^{\prime \prime}}\right\}>\beta .
$$

A necessary and sufficient condition for a function to be in the class $A_{\gamma}^{\lambda, \mu, \nu}(n, \beta)$ is obtained. Also, our paper includes linear combination, integral operators and we introduce the subclass $A_{\gamma, c_{m}}^{\lambda, \mu}(1, \beta)$ consisting of functions with negative and fixed finitely many coefficients. We study some interesting properties of $A_{\gamma, c_{m}}^{\lambda, \mu}(1, \beta)$.
\end{abstract}

2010 Mathematics Subject Classification: 30C45, 30C50.

Key words and phrases: Generalized Ruscheweyh derivatives, Fractional derivative, Integral operators, Linear combination, Radii of starlikeness, Extreme points, Weighted mean and Arithmetic mean.

\footnotetext{
${ }^{1}$ Received 8 February, 2010

Accepted for publication (in revised form) 12 July, 2010
} 


\section{Introduction}

Let $\Omega$ denote the class of functions analytic in the unit disk $U=\{z \in \mathbb{C}:|z|<1\}$ and let $A(n)$ denote the subclass of $\Omega$ consisting of functions of the form

$$
f(z)=z-\sum_{k=n+1}^{\infty} a_{k} z^{k}, \quad\left(a_{k} \geq 0, n \in \mathbb{N}=\{1,2,3, \cdots\}\right)
$$

where $f(z)$ is analytic and univalent in the unit disk $U$. Then the function $f(z) \in$ $A(n)$ is said to be in the class $S(n, \alpha)$ if and only if

$$
\operatorname{Re}\left\{\frac{z f^{\prime}(z)}{f(z)}\right\}>\alpha, \quad(z \in U, 0 \leq \alpha<1) .
$$

A function $f(z) \in S(n, \alpha)$ is called starlike function of order $\alpha$. A function $f(z) \in$ $A(n)$ is said to be in the class $C(n, \alpha)$ if and only if

$$
\operatorname{Re}\left\{1+\frac{z f^{\prime \prime}(z)}{f^{\prime}(z)}\right\}>\alpha, \quad(z \in U, 0 \leq \alpha<1) .
$$

A function $f(z) \in C(n, \alpha)$ is called convex function of order $\alpha$. It is observed that $f(z) \in C(n, \alpha)$ if and only if $z f^{\prime}(z) \in S(n, \alpha) \forall n \in\{1,2, \cdots\} \quad[9]$ A function $f(z) \in A(n)$ is said to be in the class $K(n, \alpha)$ if there is a convex function $g(z)$ such that

$$
\operatorname{Re}\left\{\frac{f^{\prime}(z)}{g^{\prime}(z)}\right\}>\alpha, \quad(\forall z \in U, 0 \leq \alpha<1) .
$$

A function $f(z) \in K(n, \alpha)$ is called close-to-convex of order $\alpha$.

We shall need the fractional derivative operator([18], [19]) in this paper.

Let $a, b, c \in \mathbb{C}$ with $c \neq 0,-1,-2, \cdots$. The Gaussian hypergeometric function ${ }_{2} F_{1}$ is defined by

$$
{ }_{2} F_{1} \equiv{ }_{2} F_{1}(a, b ; c ; z)=\sum_{n=0}^{\infty} \frac{(a)_{n}(b)_{n}}{(c)_{n}} \frac{z^{n}}{n !},
$$

where $(\lambda)_{n}$ is the Pochhammer symbol defined, in terms of the Gamma function, by

$$
(\lambda)_{n}=\frac{\Gamma(\lambda+n)}{\Gamma(\lambda)}= \begin{cases}1 & (n=0) \\ \lambda(\lambda+1) \cdots(\lambda+n-1) & (n \in \mathbb{N}) .\end{cases}
$$

Definition 1 Let $0 \leq \lambda<1$ and $\mu, \nu \in \mathbb{R}$. Then, in terms of familiar (Gauss's) hypergeometric function ${ }_{2} F_{1}$, the generalized fractional derivative operator $J_{0, z}^{\lambda, \mu, \nu}$ of a function $f(z)$ is defined by:

$$
J_{0, z}^{\lambda, \mu, \nu} f(z)=\left\{\begin{array}{lr}
\frac{1}{\Gamma(1-\lambda)} \frac{d}{d z}\left\{z^{\lambda-\mu} \int_{0}^{z}(z-\xi)^{-\lambda} f(\xi) .\right. & \\
\left.2 F_{1}\left(\mu-\lambda, 1-\nu ; 1-\lambda ; 1-\frac{\xi}{z}\right) d \xi\right\} & (0 \leq \lambda<1) \\
\frac{d^{n}}{d z^{n}} J_{0, z}^{\lambda-n, \mu, \nu} f(z), & (n \leq \lambda<n+1, n \in \mathbb{N})
\end{array}\right.
$$


where the function $f(z)$ is analytic in a simply - connected region of the z-plane containing the region, with the order

$$
f(z)=O\left(|z|^{\epsilon}\right), \quad(z \rightarrow 0)
$$

for $\epsilon>\max \{0, \mu-\nu\}-1$, and the multiplicity of $(z-\xi)^{-\lambda}$ is removed by required $\log (z-\xi)$ to be real when $z-\xi>0$.

The fractional derivative of order $\lambda$ of a function $f(z)$ is defined by

$$
D_{z}^{\lambda} f(z)=\frac{1}{\Gamma(1-\lambda)} \frac{d}{d z} \int_{0}^{z} \frac{f(\xi)}{(z-\xi)^{\lambda}} d \xi, \quad 0 \leq \lambda<1
$$

where $f(z)$ is chosen as in (1.7), and the multiplicity of $(z-\xi)^{-\lambda}$ is removed by required $\log (z-\xi)$ to be real when $z-\xi>0$.

By comparing (1.7) with (1.9), we find

$$
J_{0, z}^{\lambda, \lambda, \nu} f(z)=D_{z}^{\lambda} f(z), \quad(0 \leq \lambda<1) .
$$

In terms of gamma function, we have

$$
J_{0, z}^{\lambda, \mu, \nu} z^{k}=\frac{\Gamma(k+1) \Gamma(1-\mu+\nu+k)}{\Gamma(1-\mu+k) \Gamma(1-\lambda+\nu+k)} z^{k-\mu}
$$

$(0 \leq \lambda<1, \mu, \nu \in \mathbb{R}$ and $k>\max \{0, \mu-\nu\}-1)$.

Definition 2 Let $f(z) \in A(n)$ be given by (1.1). Then the class $A_{\gamma}^{\lambda, \mu, \nu}(n, \beta)$ is defined by

$$
\begin{gathered}
A_{\gamma}^{\lambda, \mu, \nu}(n, \beta)=\left\{f \in A(n): \operatorname{Re}\left\{\frac{z\left(\mathbf{J}_{1}^{\lambda, \mu} f(z)\right)^{\prime}}{(1-\gamma) \mathbf{J}_{1}^{\lambda, \mu} f(z)+\gamma z^{2}\left(\mathbf{J}_{1}^{\lambda, \mu} f(z)\right)^{\prime \prime}}\right\}>\beta,\right. \\
(z \in U, 0 \leq \gamma<1, n \in \mathbb{N} ; 0 \leq \beta<1 ; \lambda>-1)
\end{gathered}
$$

where $\mathbf{J}_{1}^{\lambda, \mu} f(z)$ is a generalized Ruscheweyh derivative defined as

$$
\mathbf{J}_{1}^{\lambda, \mu} f(z)=\frac{\Gamma(\mu-\lambda+\nu-2)}{\Gamma(\nu+2) \Gamma(\mu+1)} z J_{0, z}^{\lambda, \mu, \nu}\left(z^{\mu-1} f(z)\right)=z-\sum_{k=n+1}^{\infty} a_{k} C_{1}^{\lambda, \mu}(k) z^{k}
$$

where

$$
C_{1}^{\lambda, \mu}(k)=\frac{\Gamma(k+\mu) \Gamma(\nu+2+\mu-\lambda) \Gamma(k+\nu+1)}{\Gamma(k) \Gamma(k+\nu+1+\mu-\lambda) \Gamma(\nu+2) \Gamma(1+\mu)} .
$$

For $\mu=\lambda=\alpha, \nu=1$, these generalized Ruscheweyh derivatives reduce to ordinary Ruscheweyh derivatives of $f(z)$ of order $\alpha$ [14]:

$$
D^{\alpha} f(z)=\frac{z}{\Gamma(\alpha+1)} D^{\alpha}\left(z^{\alpha-1} f(z)\right)=z-\sum_{k=n+1}^{\infty} a_{k} C_{k}(\alpha) z^{k}
$$


where

$$
C_{k}(\alpha)=\frac{(\alpha+1)(\alpha+2) \cdots(\alpha+k-1)}{(k-1) !}
$$

The class $A_{\gamma}^{\lambda, \mu, \nu}(n, \beta)$ contains many well-known classes of analytic functions, for example,

(i) If $\mu=\lambda=\alpha, \nu=1, n=1$, we get the class $A_{\gamma}^{\lambda, \lambda, 1}(1, \beta)$ studied by

Tehranchi and Kulkarni [20].

(ii) If $\mu=\lambda=0, \nu=1, \alpha=\beta, \gamma=0$, we get the class of starlike functions of order $\alpha,(S(n, \alpha))$.

The same properties have been found for other classes in $[2,3,4,5,6,7,8,10,16,17]$.

Lemma 1 .[1] Let $w=u+i v$. Then

$$
\text { Re } w \geq \beta \text { if and only if }|w-(1+\beta)| \leq|w+(1-\beta)| .
$$

\section{Coefficient bounds}

The following theorem gives a necessary and sufficient condition for function to be in $A_{\gamma}^{\lambda, \mu, \nu}(n, \beta)$.

Theorem 1 Let $f(z) \in A(n)$. Then $f(z) \in A_{\gamma}^{\lambda, \mu, \nu}(n, \beta)$ if and only if

$$
\sum_{k=n+1}^{\infty}\left(\gamma \beta\left(1+k-k^{2}\right)+k-\beta\right) C_{1}^{\lambda, \mu}(k) a_{k}<1-\beta(1-\gamma)
$$

where $0 \leq \gamma<1,0 \leq \beta<1, \lambda>-1, n \in \mathbb{N}$ and $C_{1}^{\lambda, \mu}(k)$ is given by (1.14).

Proof. Assume that $f \in A_{\gamma}^{\lambda, \mu, \nu}(n, \beta)$ so we have

$$
\operatorname{Re}\left\{\frac{z\left(\mathbf{J}_{1}^{\lambda, \mu} f(z)\right)^{\prime}}{(1-\gamma) \mathbf{J}_{1}^{\lambda, \mu} f(z)+\gamma z^{2}\left(\mathbf{J}_{1}^{\lambda, \mu} f(z)\right)^{\prime \prime}}\right\}>\beta
$$

$\operatorname{Re}\left\{\frac{z-\sum_{k=n+1}^{\infty} k C_{1}^{\lambda, \mu}(k) a_{k} z^{k}}{(1-\gamma)\left(z-\sum_{k=n+1}^{\infty} C_{1}^{\lambda, \mu}(k) a_{k} z^{k}\right)+\gamma\left(-\sum_{k=n+1}^{\infty} k(k-1) C_{1}^{\lambda, \mu}(k) a_{k} z^{k}\right)}\right\}>\beta$.

Hence

$$
\operatorname{Re}\left\{\frac{1-\sum_{k=n+1}^{\infty} k C_{1}^{\lambda, \mu}(k) a_{k} z^{k}}{(1-\gamma)-\sum_{k=n+1}^{\infty}\left(1-\gamma+\gamma k(k-1) C_{1}^{\lambda, \mu}(k) a_{k} z^{k-1}\right.}\right\}>\beta .
$$


or equivalently

$$
\begin{array}{r}
\operatorname{Re}\left(\left[1-\sum_{k=n+1}^{\infty} k C_{1}^{\lambda, \mu}(k) a_{k} z^{k-1}-\beta(1-\gamma)\right.\right. \\
\left.+\beta \sum_{k=n+1}^{\infty}(1-\gamma+\gamma k(k-1)) C_{1}^{\lambda, \mu}(k) a_{k} z^{k-1}\right] /[(1-\gamma) \\
\left.\left.-\sum_{k=n+1}^{\infty}(1-\gamma+\gamma k(k-1)) C_{1}^{\lambda, \mu}(k) a_{k} z^{k-1}\right]\right)>0 .
\end{array}
$$

This inequality is correct for all $z \in U$. Letting $z \longrightarrow 1^{-}$yields

$$
\operatorname{Re}\left\{\frac{1-\beta(1-\gamma)-\sum_{k=n+1}^{\infty}(k-\beta(1-\gamma+\gamma k(k-1))) C_{1}^{\lambda, \mu}(k) a_{k}}{(1-\gamma)-\sum_{k=n+1}^{\infty}(1-\gamma+\gamma k(k-1)) C_{1}^{\lambda, \mu}(k) a_{k}}\right\}>0
$$

and so by the mean value theorem, we have

$$
\operatorname{Re}\left\{1-\beta(1-\gamma)-\sum_{k=n+1}^{\infty}\left(k-\beta\left(1-\gamma+\gamma k^{2}-\gamma k\right)\right) C_{1}^{\lambda, \mu}(k) a_{k}\right\}>0,
$$

so we have

$$
\sum_{k=n+1}^{\infty}\left(\gamma \beta\left(1+k-k^{2}\right)+k-\beta\right) C_{1}^{\lambda, \mu}(k) a_{k}<1-\beta(1-\gamma) .
$$

Conversely, let (2.1) hold. We will prove that (1.12) is correct and then $f \in$ $A_{\gamma}^{\lambda, \mu, \nu}(n, \beta)$. By Lemma 1 it is enough to prove that $|w-(1+\beta)|<|w+(1-\beta)|$ where

$$
w=\frac{z\left(\mathbf{J}_{1}^{\lambda, \mu} f(z)\right)^{\prime}}{(1-\gamma) \mathbf{J}_{1}^{\lambda, \mu} f(z)+\gamma z^{2}\left(\mathbf{J}_{1}^{\lambda, \mu} f(z)\right)^{\prime \prime}}
$$

or show that

$$
\begin{aligned}
T & =\frac{1}{|N(z)|}\left|z\left(\mathbf{J}_{1}^{\lambda, \mu} f(z)\right)^{\prime}-(1+\beta)(1-\gamma) \mathbf{J}_{1}^{\lambda, \mu} f(z)-(1+\beta) \gamma z^{2}\left(\mathbf{J}_{1}^{\lambda, \mu} f(z)\right)^{\prime \prime}\right| \\
& <\frac{1}{|N(z)|}\left|z\left(\mathbf{J}_{1}^{\lambda, \mu} f(z)\right)^{\prime}+(1-\beta)(1-\gamma) \mathbf{J}_{1}^{\lambda, \mu} f(z)+(1-\beta) \gamma z^{2}\left(\mathbf{J}_{1}^{\lambda, \mu} f(z)\right)^{\prime \prime}\right| \\
& =Q
\end{aligned}
$$

where $N(z)=(1-\gamma) \mathbf{J}_{1}^{\lambda, \mu} f(z)+\gamma z^{2}\left(\mathbf{J}_{1}^{\lambda, \mu} f(z)\right)^{\prime \prime}$ and it is easy to verify that $Q-T>0$ and so the proof is complete.

Corollary 1 Let $f \in A_{\gamma}^{\lambda, \mu, \nu}(n, \beta)$. Then

$$
a_{k}<\frac{1-\beta(1-\gamma)}{C_{1}^{\lambda, \mu}(k)\left|\gamma \beta\left(1+k-k^{2}\right)+k-\beta\right|}, \quad k=n+1, n+2, \cdots .
$$




\section{Linear combination}

In the following theorem, we will show that this class $A_{\gamma}^{\lambda, \mu, \nu}(n, \beta)$ is closed under linear combination.

Theorem 2 Let $f_{i}(z)=z-\sum_{k=n+1}^{\infty} a_{k, i} z^{k} \in A_{\gamma}^{\lambda, \mu, \nu}(n, \beta), i \in\{1,2, \cdots, t\}$ and $0<C_{i}<1$ such that

$$
\sum_{i=1}^{t} C_{i}=1
$$

Then the function $G(z)$ defined by $G(z)=\sum_{i=1}^{t} C_{i} f_{i}(z)$ is also in $A_{\gamma}^{\lambda, \mu, \nu}(n, \beta)$.

Proof. For every $i \in\{1,2, \cdots, t\}$ we obtain

$$
\sum_{k=n+1}^{\infty} \frac{\left(\gamma \beta\left(1+k-k^{2}\right)+k-\beta\right) C_{1}^{\lambda, \mu}(k)}{1-\beta(1-\gamma)} a_{k, i}<1 .
$$

Since

$$
G(z)=\sum_{i=1}^{t} C_{i} f_{i}(z)=\sum_{i=1}^{t} C_{i}\left(z-\sum_{k=n+1}^{\infty} a_{k, i} z^{k}\right)=z-\sum_{k=n+1}^{\infty}\left(\sum_{i=1}^{t} C_{i} a_{k, i}\right) z^{k} .
$$

Therefore,

$$
\begin{gathered}
\sum_{k=n+1}^{\infty} \frac{\left(\gamma \beta\left(1+k-k^{2}\right)+k-\beta\right) C_{1}^{\lambda, \mu}(k)}{1-\beta(1-\gamma)}\left[\sum_{i=1}^{t} C_{i} a_{k, i}\right] \\
=\sum_{i=1}^{t} C_{i}\left[\sum_{k=n+1}^{\infty} \frac{\left(\gamma \beta\left(1+k-k^{2}\right)+k-\beta\right) C_{1}^{\lambda, \mu}(k)}{1-\beta(1-\gamma)} a_{k, i}\right]<\sum_{i=1}^{t} C_{i}=1 .
\end{gathered}
$$

Hence $G(z) \in A_{\gamma}^{\lambda, \mu, \nu}(n, \beta)$ and therefore the proof is complete.

\section{Integral operators}

In the next theorems, we obtain some results by using the integral operators for this class.

Theorem 3 Let $f(z) \in A_{\gamma}^{\lambda, \mu, \nu}(n, \beta)$, Komato operator [11] of $f$ is defined by

$$
y(z)=\int_{0}^{1} \frac{(d+1)^{\theta}}{\Gamma(\theta)} \ell^{d}\left(\log \frac{1}{\ell}\right)^{\theta-1} \frac{f(\ell z)}{\ell} d \ell, d>-1, \theta \geq 0 .
$$

Then $y(z) \in A_{\gamma}^{\lambda, \mu, \nu}(n, \beta)$. 
Proof. We have $\int_{0}^{1} \ell^{d}\left(\log \frac{1}{\ell}\right)^{\theta-1} d \ell=\frac{\Gamma(\theta)}{(d+1)^{\theta}}$ and $\int_{0}^{1} \ell^{k+d-1}\left(\log \frac{1}{\ell}\right)^{\theta-1} d \ell=\frac{\Gamma(\theta)}{(d+k)^{\theta}}$, $k=n+1, n+2, \cdots$, then we obtain

$$
\begin{gathered}
y(z)=\frac{(d+1)^{\theta}}{\Gamma(\theta)}\left[\int_{0}^{1} \ell^{d}\left(\log \frac{1}{\ell}\right)^{\theta-1} z d \ell-\sum_{k=n+1}^{\infty} z^{k} \int_{0}^{1} a_{k} \ell^{d+k-1}\left(\log \frac{1}{\ell}\right)^{\theta-1} d \ell\right] \\
=z-\sum_{k=n+1}^{\infty}\left(\frac{d+1}{d+k}\right)^{\theta} a_{k} z^{k} .
\end{gathered}
$$

Since $f \in A_{\gamma}^{\lambda, \mu, \nu}(n, \beta)$ and since $\left(\frac{d+1}{d+k}\right)^{\theta}<1$ for $k=n+1, n+2, \cdots$, then

$$
\sum_{k=n+1}^{\infty}\left[\frac{\gamma \beta\left(1+k-k^{2}\right)+k-\beta}{1-\beta(1-\gamma)}\right]\left(\frac{d+1}{d+k}\right)^{\theta} a_{k} C_{1}^{\lambda, \mu}(k)<1 .
$$

This completes the proof.

Theorem 4 The function $y(z)$ defined in (4.1) is starlike of order $\eta$ in $|z|<$ $r_{1}(\lambda, \mu, \nu, \gamma, \beta, d, \theta, \eta)$, where

$$
\begin{gathered}
r_{1}(\lambda, \mu, \nu, \gamma, \beta, d, \theta, \eta)= \\
\inf _{k}\left\{\frac{1-\eta}{k-\eta}\left(\frac{d+k}{d+1}\right)^{\theta}\left[\frac{\gamma \beta\left(1+k-k^{2}\right)+k-\beta}{1-\beta(1-\gamma)}\right] C_{1}^{\lambda, \mu}(k)\right\}^{\frac{1}{k-1}} .
\end{gathered}
$$

Proof. We must show that

$$
\left|\frac{z y^{\prime}(z)}{y(z)}-1\right|<1-\eta
$$

But

$$
\begin{aligned}
\left|\frac{z y^{\prime}(z)}{y(z)}-1\right| & =\left|\frac{z-\sum_{k=n+1}^{\infty} k\left(\frac{d+1}{d+k}\right)^{\theta} a_{k} z^{k}}{z-\sum_{k=n+1}^{\infty}\left(\frac{d+1}{d+k}\right)^{\theta} a_{k} z^{k}}-1\right| \\
& =\left|\frac{\sum_{k=n+1}^{\infty}(k-1)\left(\frac{d+1}{d+k}\right)^{\theta} a_{k} z^{k-1}}{1-\sum_{k=n+1}^{\infty}\left(\frac{d+1}{d+k}\right)^{\theta} a_{k} z^{k-1}}\right|
\end{aligned}
$$




$$
\leq \frac{\sum_{k=n+1}^{\infty}(k-1)\left(\frac{d+1}{d+k}\right)^{\theta} a_{k}|z|^{k-1}}{1-\sum_{k=n+1}^{\infty}\left(\frac{d+1}{d+k}\right)^{\theta} a_{k}|z|^{k-1}}<1-\eta
$$

Then

$$
\sum_{k=n+1}^{\infty}(k-1)\left(\frac{d+1}{d+k}\right)^{\theta} a_{k}|z|^{k-1}<1-\eta-(1-\eta) \sum_{k=n+1}^{\infty}\left(\frac{d+1}{d+k}\right)^{\theta} a_{k}|z|^{k-1} .
$$

Hence

$$
\sum_{k=n+1}^{\infty} \frac{k-\eta}{1-\eta}\left(\frac{d+1}{d+k}\right)^{\theta} a_{k}|z|^{k-1}<1 .
$$

Now in view of (2.1), therefore (4.2) holds if

$$
|z|^{k-1}<\left(\frac{1-\eta}{k-\eta}\right)\left(\frac{d+k}{d+1}\right)^{\theta}\left[\frac{\gamma \beta\left(1+k-k^{2}\right)+k-\beta}{1-\beta(1-\gamma)}\right] C_{1}^{\lambda, \mu}(k) .
$$

This completes the proof.

Corollary 2 The function $y(z)$ defined in Theorem 3 is convex of order $\eta$ in $|z|<$ $r_{2}(\lambda, \mu, \nu, \gamma, \beta, d, \theta, \eta)$, where

$$
\begin{gathered}
r_{2}(\lambda, \mu, \nu, \gamma, \beta, d, \theta, \eta) \\
=\inf _{k}\left\{\frac{1-\eta}{k(k-\eta)}\left(\frac{d+k}{d+1}\right)^{\theta}\left[\frac{\gamma \beta\left(1+k-k^{2}\right)+k-\beta}{1-\beta(1-\gamma)}\right] C_{1}^{\lambda, \mu}(k)\right\}^{\frac{1}{k-1}} .
\end{gathered}
$$

Theorem 5 Let $f \in A_{\gamma}^{\lambda, \mu, \nu}(n, \beta)$. Then the function $T_{\psi}(z)$ defined by $T_{\psi}(z)=(1-\psi) z+\psi \int_{0}^{z} \frac{f(\ell)}{\ell} d \ell, \psi \geq 0, z \in U$ is in $A_{\gamma}^{\lambda, \mu, \nu}(n, \beta)$ if $0 \leq \psi \leq n+1$.

\section{Proof.}

$$
\begin{gathered}
T_{\psi}(z)=(1-\psi) z+\psi \int_{0}^{z}\left(\frac{\ell-\sum_{k=n+1}^{\infty} a_{k} \ell^{k}}{\ell}\right) d \ell \\
=z-\psi z+\psi\left(\ell-\left.\sum_{k=n+1}^{\infty} a_{k}\left(\frac{1}{k} \ell^{k}\right)\right|_{0} ^{z}\right) \\
=z-\psi z+\psi z-\sum_{k=n+1}^{\infty} a_{k} \frac{\psi}{k} z^{k}=z-\sum_{k=n+1}^{\infty} a_{k} \frac{\psi}{k} z^{k},
\end{gathered}
$$


but

$$
\begin{aligned}
& \sum_{k=n+1}^{\infty}\left[\frac{\gamma \beta\left(1+k-k^{2}\right)+k-\beta}{1-\beta(1-\gamma)}\right] \frac{\psi a_{k}}{k} C_{1}^{\lambda, \mu}(k) \\
\leq & \sum_{k=n+1}^{\infty}\left[\frac{\gamma \beta\left(1+k-k^{2}\right)+k-\beta}{1-\beta(1-\gamma)}\right] \frac{\psi a_{k}}{n+1} C_{1}^{\lambda, \mu}(k)<1 .
\end{aligned}
$$

This inequality completes the proof.

Remark 1 By the similar proofs which we applied for Theorem 4 and Corollary 2, we conclude that the function $T_{\psi}(z)$ is starlike of order $\eta(0 \leq \eta<1)$ in $|z|<r_{1}\left(\mu, \nu, \gamma,{ }_{2} \psi, \eta\right)$, where

$$
r_{1}(\lambda, \mu, \nu, \gamma, \beta, \psi, \eta)=\inf _{k}\left\{\frac{k(1-\eta)}{\psi(k-\eta)}\left[\frac{\gamma \beta\left(1+k-k^{2}\right)+k-\beta}{1-\beta(1-\gamma)}\right] C_{1}^{\lambda, \mu}(k)\right\}^{\frac{1}{k-1}}
$$

Also $T_{\psi}(z)$ is convex of order $\eta(0 \leq \eta<1)$ in $|z|<r_{2}(\lambda, \mu, \nu, \gamma, \beta, \psi, \eta)$, where

$$
r_{2}(\lambda, \mu, \nu, \gamma, \beta, \psi, \eta)=\inf _{k}\left\{\frac{(1-\eta)}{\psi(k-\eta)}\left[\frac{\gamma \beta\left(1+k-k^{2}\right)+k-\beta}{1-\beta(1-\gamma)}\right] C_{1}^{\lambda, \mu}(k)\right\}^{\frac{1}{k-1}}
$$

Theorem 6 (i) Let $f \in A_{\gamma}^{\lambda, \mu, \nu}(n, \beta)$. Then for every $\delta \geq 0$ the function $F_{\delta}(z)$ defined by $F_{\delta}(z)=(1-\delta) f(z)+\delta \int_{0}^{z} \frac{f(\ell)}{\ell} d \ell$ also belongs to $A_{\gamma}^{\lambda, \mu, \nu}(n, \beta)$.

(ii) If $f \in A_{0}^{0,0,1}(n, \beta)=S(n, \beta), \frac{f(z)}{z} \neq 0, z \in U$, also $0 \leq \delta<1$, then $F_{\delta}(z)$ is close-to-convex of order $\delta$.

Proof. (i)

$$
\begin{gathered}
F_{\delta}(z)=(1-\delta)\left(z-\sum_{k=n+1}^{\infty} a_{k} z^{k}\right)+\delta \int_{0}^{z} \frac{\ell-\sum_{k=n+1}^{\infty} a_{k} \ell^{k}}{\ell} d \ell \\
=z-\delta z-(1-\delta) \sum_{k=n+1}^{\infty} a_{k} z^{k}+\delta z-\sum_{k=n+1}^{\infty} a_{k} \frac{\delta}{k} z^{k} \\
=z-\sum_{k=n+1}^{\infty}\left(1-\delta+\frac{\delta}{k}\right) a_{k} z^{k} .
\end{gathered}
$$

By $(2.1)$ and this fact that $1-\delta+\frac{\delta}{k}=1-\left(\delta-\frac{\delta}{k}\right)<1$ for $k \geq n+1$, we have

$$
\sum_{k=n+1}^{\infty} \frac{\gamma \beta\left(1+k-k^{2}\right)+k-\beta}{1-\beta(1-\gamma)}\left(1-\delta+\frac{\delta}{k}\right) a_{k} C_{1}^{\lambda, \mu}(k)<1
$$


So we obtain $F_{\delta}(z) \in A_{\gamma}^{\lambda, \mu, \nu}(n, \beta)$.

(ii) Since $F_{\delta}^{\prime}(z)=(1-\delta) f^{\prime}(z)+\delta \frac{f(z)}{z}$, so $\frac{z F_{\delta}^{\prime}(z)}{f(z)}=\delta+(1-\delta) \frac{z f^{\prime}(z)}{f(z)}$ and

$$
\operatorname{Re}\left\{\frac{z F_{\delta}^{\prime}(z)}{f(z)}\right\}=\delta+(1-\delta) \operatorname{Re}\left\{\frac{z f^{\prime}(z)}{f(z)}\right\}>\delta
$$

and this by (1.5) shows that $F_{\delta}(z)$ is close-to-convex of order $\delta$.

Remark 2 The function $F_{\delta}(z)$ defined in Theorem 6 is starlike of order $\phi(0 \leq \phi<$ 1) in $|z|<r_{1}(\lambda, \mu, \nu, \gamma, \beta, \delta, \phi)$, where

$$
r_{1}(\lambda, \mu, \nu, \gamma, \beta, \delta, \phi)=\inf _{k}\left\{\frac{(1-\phi)\left[\gamma \beta\left(1+k-k^{2}\right)+k-\beta\right]}{(k-\phi)\left(1-\delta+\frac{\delta}{k}\right)[1-\beta(1-\gamma)]} C_{1}^{\lambda, \mu}(k)\right\}^{\frac{1}{k-1}} .
$$

Also $F_{\delta}(z)$ is convex of order $\phi(0 \leq \phi<1)$ in $|z|<r_{2}(\lambda, \mu, \nu, \gamma, \beta, \delta, \phi)$, where

$$
r_{2}(\lambda, \mu, \nu, \gamma, \beta, \delta, \phi)=\inf _{k}\left\{\frac{(1-\phi)\left[\gamma \beta\left(1+k-k^{2}\right)+k-\beta\right]}{k(k-\phi)\left(1-\delta+\frac{\delta}{k}\right)[1-\beta(1-\gamma)]} C_{1}^{\lambda, \mu}(k)\right\}^{\frac{1}{k-1}}
$$

\section{Some properties of a subclass $A_{\gamma, c_{m}}^{\mu, \nu}(1, \beta)$}

We introduce the class $A_{\gamma, c_{m}}^{\lambda, \mu, \nu}(1, \beta)$ the subclass of $A_{\gamma}^{\lambda, \mu, \nu}(1, \beta)$ where

$$
A_{\gamma}^{\lambda, \mu, \nu}(1, \beta)=\left\{f \in A(1): \operatorname{Re}\left\{\frac{z\left(\mathbf{J}_{1}^{\lambda, \mu} f(z)\right)^{\prime}}{(1-\gamma) \mathbf{J}_{1}^{\lambda, \mu} f(z)+\gamma z^{2}\left(\mathbf{J}_{1}^{\lambda, \mu} f(z)\right)^{\prime \prime}}\right\}>\beta\right\},
$$

consisting of functions with negative and fixed finitely many coefficients of the form

$$
f(z)=z-\sum_{i=2}^{m} \frac{(1-\beta(1-\gamma)) C_{i}}{\left[\gamma \beta\left(1+i-i^{2}\right)+i-\beta\right] C_{1}^{\lambda, \mu}(i)} z^{i}-\sum_{k=m+1}^{\infty} a_{k} z^{k},
$$

where $m=2,3, \cdots, a_{k} \geq 0$ for $k=m+1, m+2, \cdots, 0 \leq C_{i} \leq 1$ for $i=2,3, \cdots, m$ and $0 \leq \sum_{i=2}^{m} c_{i} \leq 1$.

The different cases were studied earlier by many authors e.g. [12], [13], [15].

We need the following lemma which has been proved in general case in Theorem 1 .

Lemma 2 Let $f(z)=z-\sum_{k=2}^{\infty} a_{k} z^{k} \in A(1)$. Then $f \in A_{\gamma}^{\lambda, \mu, \nu}(1, \beta)$ if and only if

$$
\sum_{k=2}^{\infty} \frac{\gamma \beta\left(1+k-k^{2}\right)+k-\beta}{1-\beta(1-\gamma)} C_{1}^{\lambda, \mu}(k) a_{k}<1 .
$$

The following theorem gives a necessary and sufficient condition for a function to be in $A_{\gamma, c_{m}}^{\lambda, \mu, \nu}(1, \beta)$. 
Theorem 7 Let $f(z)$ be defined by (5.1). Then $f \in A_{\gamma, c_{m}}^{\lambda, \mu, \nu}(1, \beta)$ if and only if

$$
\sum_{k=m+1}^{\infty} \frac{\gamma \beta\left(1+k-k^{2}\right)+k-\beta}{1-\beta(1-\gamma)} C_{1}^{\lambda, \mu}(k) a_{k}<1-\sum_{i=2}^{m} c_{i} .
$$

Proof. By letting

$$
a_{i}=\frac{(1-\beta(1-\gamma)) c_{i}}{\left[\gamma \beta\left(1+i-i^{2}\right)+i-\underline{]} C_{1}^{\lambda, \mu}(i)\right.}
$$

since $A_{\gamma, c_{m}}^{\lambda, \mu, \nu}(1, \beta) \subset A_{\gamma}^{\lambda, \mu, \nu}(1, \beta)$ so $f \in A_{\gamma, c_{m}}^{\lambda, \mu, \nu}(1, \beta)$ if and only if

$$
\sum_{i=2}^{m} \frac{\gamma \beta\left(1+i-i^{2}\right)+i-\beta}{1-\beta(1-\gamma)} a_{i} C_{1}^{\lambda, \mu}(i)+\sum_{k=m+1}^{\infty} \frac{\gamma \beta\left(1+k-k^{2}\right)+k-\beta}{1-\beta(1-\gamma)} a_{k} C_{1}^{\lambda, \mu}(k)<1
$$

or

$$
\sum_{k=m+1}^{\infty} \frac{\gamma \beta\left(1+k-k^{2}\right)+k-\beta}{1-\beta(1-\gamma)} a_{k} C_{1}^{\mu}(k)<1-\sum_{i=2}^{m} c_{i}
$$

and the proof is complete.

Corollary 3 If $f(z)$ defined by (5.1) be in $A_{\gamma, c_{m}}^{\lambda, \mu, \nu}(1, \beta)$, then for $k \geq m+1$, we have

$$
a_{k} \leq \frac{(1-\beta(1-\gamma))\left(1-\sum_{i=2}^{m} c_{i}\right)}{\left[\gamma \beta\left(1+k-k^{2}\right)+k-\beta\right] C_{1}^{\lambda, \mu}(k)} .
$$

This result is sharp due to the function $f(z)$ defined by

$$
\begin{aligned}
f(z) & =z-\sum_{i=2}^{m} \frac{(1-\beta(1-\gamma)) c_{i}}{\left[\gamma \beta\left(1+i-i^{2}\right)+i-\beta\right] C_{1}^{\lambda, \mu}(i)} z^{i} \\
& -\frac{(1-\beta(1-\gamma))\left(1-\sum_{i=2}^{m} c_{i}\right)}{\left[\gamma \beta\left(1+k-k^{2}\right)+k-\beta\right] C_{1}^{\lambda, \mu}(k)} z^{k} .
\end{aligned}
$$

\section{Theorem 8 Let}

$$
f_{j}(z)=z-\sum_{i=2}^{m} \frac{(1-\beta(1-\gamma)) c_{i}}{\left(\gamma \beta\left(1+i-i^{2}\right)+i-\beta\right) C_{1}^{\lambda, \mu}(i)} z^{i}-\sum_{k=m+1}^{\infty} a_{k, j} z^{k}
$$

for $j=1,2, \cdots, \iota$ be in $A_{\gamma, c_{m}}^{\lambda, \mu, \nu}(1, \beta)$. Then the function $F(z)=\sum_{j=1}^{\iota} \eta_{j} f_{j}(z)$ is also in $A_{\gamma, c_{m}}^{\lambda, \mu, \nu}(1, \beta)$ where $\sum_{j=1}^{\iota} \eta_{j}=1,0 \leq \sum_{i=2}^{m} c_{i} \leq 1,0 \leq c_{i} \leq 1$.

Proof. By Theorem 7 for every $j=1,2, \cdots, \iota$, we have

$$
\sum_{k=m+1}^{\infty} \frac{\gamma \beta\left(1+k-k^{2}\right)+k-\beta}{1-\beta(1-\gamma)} a_{k, j} C_{1}^{\lambda, \mu}(k)<1-\sum_{i=2}^{m} c_{i} .
$$


But

$$
\begin{aligned}
F(z)=\sum_{j=1}^{\iota} \eta_{j} f_{j}(z) & =z-\sum_{i=2}^{m} \frac{(1-\beta(1-\gamma)) c_{i}}{\left(\gamma \beta\left(1+i-i^{2}\right)+i-\beta\right) C_{1}^{\lambda, \mu}(i)} z^{i} \\
& -\sum_{k=m+1}^{\infty}\left(\sum_{j=1}^{\iota} \eta_{j} a_{k, j}\right) z^{k} .
\end{aligned}
$$

So

$$
\begin{gathered}
\sum_{k=m+1}^{\infty} \frac{\gamma \beta\left(1+k-k^{2}\right)+k-\beta}{1-\beta(1-\gamma)} C_{1}^{\lambda, \mu}(k)\left(\sum_{j=1}^{\iota} \eta_{j} a_{k, j}\right) \\
=\sum_{j=1}^{\iota} \sum_{k=m+1}^{\infty}\left(\frac{\gamma \beta\left(1+k-k^{2}\right)+k-\beta}{1-\beta(1-\gamma)} C_{1}^{\lambda, \mu}(k) a_{k, j}\right) \eta_{j} \\
<\sum_{j=1}^{\iota}\left(1-\sum_{i=2}^{m} c_{i}\right) \eta_{j}=1-\sum_{i=2}^{m} c_{i}
\end{gathered}
$$

and the proof is complete.

Remark 3 If $f_{1}(z), f_{2}(z)$ be in $A_{\gamma, c_{m}}^{\lambda, \mu, \nu}(1, \beta)$, then the function $H(z)=\frac{1}{2}\left(f_{1}(z)+f_{2}(z)\right)$ is also in $A_{\gamma, c_{m}}^{\lambda, \mu, \nu}(1, \beta)$.

Remark 4 The class $A_{\gamma, c_{m}}^{\lambda, \mu, \nu}(1, \beta)$ is a convex set.

In the next theorem, we will prove the arithmetic mean property.

Theorem 9 Let $f_{j}(z),(j=1,2, \cdots, \iota)$ defined by (5.3) be in $A_{\gamma, c_{m}}^{\lambda, \mu, \nu}(1, \beta)$. Then the function

$$
V(z)=z-\sum_{i=2}^{m} \frac{(1-\beta(1-\gamma)) c_{i}}{\left(\gamma \beta\left(1+i-i^{2}\right)+i-\beta\right) C_{1}^{\lambda, \mu}(i)} z^{i}-\sum_{k=m+1}^{\infty} b_{k} z^{k},\left(b_{k} \geq 0\right)
$$

is also in $A_{\gamma, c_{m}}^{\lambda, \mu, \nu}(1, \beta)$, where $b_{k}=\frac{1}{\iota} \sum_{j=1}^{\iota} a_{k, j}$.

Proof. We have

$$
\begin{aligned}
& \sum_{k=m+1}^{\infty} \frac{\gamma \beta\left(1+k-k^{2}\right)+k-\beta}{1-\beta(1-\gamma)} b_{k} C_{1}^{\lambda, \mu}(k) \\
= & \sum_{k=m+1}^{\infty} \frac{\gamma \beta\left(1+k-k^{2}\right)+k-\beta}{\iota(1-\beta(1-\gamma))}\left(\sum_{j=1}^{\iota} a_{k, j}\right) C_{1}^{\lambda, \mu}(k) \\
= & \frac{1}{\iota} \sum_{j=1}^{\iota}\left[\sum_{k=m+1}^{\infty} \frac{\gamma \beta\left(1+k-k^{2}\right)+k-\beta}{1-\beta(1-\gamma)} a_{k, j} C_{1}^{\lambda, \mu}(k)\right]
\end{aligned}
$$


(by Theorem 7))

$$
<\frac{1}{\iota} \sum_{j=1}^{\iota}\left(1-\sum_{i=2}^{m} c_{i}\right)=1-\sum_{i=2}^{m} c_{i},
$$

and the proof is complete.

Definition 3 Let $f(z)$ and $g(z)$ belong to $A(n)$. Then the weighted mean $h_{j}(z)$ of $f(z)$ and $g(z)$ is given by

$$
h_{j}(z)=\frac{1}{2}[(1-j) f(z)+(1+j) g(z)] .
$$

Theorem 10 If $f(z)$ and $g(z)$ be in the class $A_{\gamma, c_{m}}^{\lambda, \mu, \nu}(1, \beta)$, then the weighted mean of $f(z)$ and $g(z)$ is also in $A_{\gamma, c_{m}}^{\lambda, \mu, \nu}(1, \beta)$.

Proof. By using Definition 3, we obtain

$$
\begin{gathered}
h_{j}(z)=\frac{1}{2}\left[(1-j)\left(z-\sum_{i=2}^{m} \frac{(1-(1-\gamma)) c_{i}}{\left[\gamma \beta\left(1+i-i^{2}\right)+i-\beta\right] C_{1}^{\lambda, \mu}(i)} z^{i}-\sum_{k=m+1}^{\infty} a_{k} z^{k}\right)\right. \\
\left.+(1+j)\left(z-\sum_{i=2}^{m} \frac{1-\beta(1-\gamma)) c_{i}}{\left[\gamma \beta\left(1+i-i^{2}\right)+i-\beta\right] C_{1}^{\lambda, \mu}(i)} z^{i}-\sum_{k=m+1}^{\infty} b_{k} z^{k}\right)\right] \\
=z-\sum_{i=2}^{m} \frac{(1-\beta(1-\gamma)) c_{i}}{\left[\gamma \beta\left(1+i-i^{2}\right)+i-\beta\right] C_{1}^{\lambda, \mu}(i)} z^{i} \\
-\sum_{k=m+1}^{\infty} \frac{1}{2}\left[(1-j) a_{k}+(1+j) b_{k}\right] z^{k} .
\end{gathered}
$$

Since $f(z)$ and $g(z)$ are in the class $A_{\gamma, c_{m}}^{\lambda, \mu, \nu}(1, \beta)$ using Theorem 8 we have

$$
\begin{aligned}
& \sum_{k=m+1}^{\infty} \frac{\left[\gamma \beta\left(1+k-k^{2}\right)+k-\beta\right]}{(1-\beta(1-\gamma))} C_{1}^{\lambda, \mu}(k) \frac{1}{2}\left[(1-j) a_{k}+(1+j) b_{k}\right] \\
& =\frac{1}{2} \sum_{k=m+1}^{\infty} \frac{\left[\gamma \beta\left(1+k-k^{2}\right)+k-\beta\right]}{(1-\beta(1-\gamma))}(1-j) C_{1}^{\lambda, \mu}(k) a_{k} \\
& \quad+\frac{1}{2} \sum_{k=m+1}^{\infty} \frac{\left[\gamma \beta\left(1+k-k^{2}\right)+k-\beta\right]}{(1-\beta(1-\gamma))}(1+j) C_{1}^{\lambda, \mu}(k) b_{k} \\
& \leq \frac{1}{2}(1-j)\left(1-\sum_{i=2}^{m} c_{i}\right)+\frac{1}{2}(1+j)\left(1-\sum_{i=2}^{m} c_{i}\right)=1-\sum_{i=2}^{m} c_{i},
\end{aligned}
$$

and again by Theorem $8, h_{j}(z) \in A_{\gamma, c_{m}}^{\lambda, \mu}(1, \beta)$.

Now we will find the extreme points of the class $A_{\gamma, c_{m}}^{\lambda, \mu, \nu}(1, \beta)$ but we need the following theorem to prove. 
Theorem 11 Let

$$
f_{m}(z)=z-\sum_{i=2}^{m} \frac{(1-\beta(1-\gamma)) c_{i}}{\left[\gamma \beta\left(1+i-i^{2}\right)+i-\beta\right] C_{1}^{\lambda, \mu}(i)} z^{i}
$$

and for $k \geq m+1$

$$
\begin{gathered}
f_{k}(z)=z-\sum_{i=2}^{m} \frac{(1-\beta(1-\gamma)) c_{i}}{\left[\gamma \beta\left(1+i-i^{2}\right)+k-\beta\right] C_{1}^{\lambda, \mu}(i)} z^{i} \\
-\frac{(1-\beta(1-\gamma))\left(1-\sum_{i=2}^{m} c_{i}\right)}{\left[\gamma \beta\left(1+k-k^{2}\right)+k-\beta\right] C_{1}^{\lambda, \mu}(k)} z^{k} .
\end{gathered}
$$

Then the function $X(z)$ is in $A_{\gamma, c_{m}}^{\lambda, \mu, \nu}(1, \beta)$ if and only if it can be expressed in the form

$$
X(z)=\sum_{k=m}^{\infty} \sigma_{k} f_{k}(z)
$$

where $\sigma_{k} \geq 0 \quad(k \geq m)$ and $\sum_{k=m}^{\infty} \sigma_{k}=1$.

Proof. Let $X(z)=\sum_{k=m}^{\infty} \sigma_{k} f_{k}(z)$. Then

$$
\begin{aligned}
& X(z)=\sigma_{m} f_{m}(z)+ \sum_{k=m+1}^{\infty} \sigma_{k} f_{k}(z)=\sigma_{m} z-\sigma_{m} \sum_{i=2}^{m} \frac{(1-\beta(1-\gamma)) c_{i}}{\left[\gamma \beta\left(1+i-i^{2}\right)+i-\beta\right] C_{1}^{\lambda, \mu}(i)} z^{i} \\
&+\sum_{k=m+1}^{\infty} \sigma_{k} z-\sum_{k=m+1}^{\infty} \sigma_{k}\left(\sum_{i=2}^{m} \frac{(1-\beta(1-\gamma)) c_{i}}{\left[\gamma \beta\left(1+i-i^{2}\right)+i-\beta\right] C_{1}^{\lambda, \mu}(i)} z^{i}\right) \\
&-\sum_{k=m+1}^{\infty} \sigma_{k}\left(\frac{(1-\beta(1-\gamma))\left(1-\sum_{i=2}^{m} c_{i}\right)}{\left[\gamma \beta\left(1+k-k^{2}\right)+k-\beta\right] C_{1}^{\lambda, \mu}(k)} z^{k}\right) \\
&=\left(\sigma_{m}+\sum_{k=m+1}^{m} \sigma_{k}\right) z-\left(\sigma_{m}+\sum_{k=m+1}^{\infty} \sigma_{k}\right) \\
& \quad \times \sum_{i=2}^{m} \frac{(1-\beta(1-\gamma)) c_{i}}{\left[\gamma\left(1+i-i^{2}\right)+i-\underline{l} C_{1}^{\lambda, \mu}(i)\right.} z^{i} \\
& \sum_{k=m+1}^{\infty} \frac{\left(1-\sum_{i=2}^{m} c_{i}\right)(1-\beta(1-\gamma))}{\left[\gamma \beta\left(1+k-k^{2}\right)+k-\beta\right] C_{1}^{\lambda, \mu}(k)} \sigma_{k} z^{k} \\
&=z-\sum_{i=2}^{m} \frac{(1-\beta(1-\gamma)) c_{i}}{\left[\gamma \beta\left(1+i-i^{2}\right)+i-\beta\right] C_{1}^{\lambda, \mu}(i)} z^{i} \\
&-\sum_{k=m+1}^{\infty} \frac{\left(1-\sum_{i=2}^{m} c_{i}\right)(1-\beta(1-\gamma)) \sigma_{k}}{\left[\gamma \beta\left(1+k-k^{2}\right)+k-\beta\right] C_{1}^{\lambda, \mu}(k)} z^{k} .
\end{aligned}
$$


Finally we have

$$
\begin{gathered}
\sum_{k=m+1}^{\infty} \frac{\left[\gamma \beta\left(1+k-k^{2}\right)+k-\beta\right]\left(1-\sum_{i=2}^{m} c_{i}\right)(1-\beta(1-\gamma)) \sigma_{k}}{\left[\gamma \beta\left(1+k-k^{2}\right)+k-\beta\right] C_{1}^{\lambda, \mu}(k)(1-\beta(1-\gamma))} C_{1}^{\lambda, \mu}(k) \\
=\left(1-\sum_{i=2}^{m} c_{i}\right) \sum_{k=m+1}^{\infty} \sigma_{k}=\left(1-\sum_{i=2}^{m} c_{i}\right)\left(1-\sigma_{m}\right)<1-\sum_{i=2}^{m} c_{i} .
\end{gathered}
$$

Thus $X(z) \in A_{\gamma, c_{m}}^{\lambda, \mu, \nu}(1, \beta)$.

Conversely, assume $X(z) \in A_{\gamma, c_{m}}^{\lambda, \mu, \nu}(1, \beta)$ so

$$
X(z)=z-\sum_{i=2}^{m} \frac{(1-\beta(1-\gamma)) c_{i}}{\left[\gamma \beta\left(1+i-i^{2}\right)+i-\underline{]} C_{1}^{\lambda, \mu}(i)\right.} z^{i}-\sum_{k=m+1}^{\infty} a_{k} z^{k} .
$$

By putting

$$
\sigma_{k}=\frac{\left[\gamma \beta\left(1+k-k^{2}\right)+k-\beta\right] C_{1}^{\lambda, \mu}(k)}{(1-\beta(1-\gamma))\left(1-\sum_{i=1}^{m} c_{i}\right)} a_{k}, \quad(k \geq m+1)
$$

we have $\sigma_{k} \geq 0$ and if we set $\sigma_{m}=1-\sum_{k=m+1}^{\infty} \sigma_{k}$, we get

$$
\begin{aligned}
X(z) & =z-\sum_{i=2}^{m} \frac{(1-\beta(1-\gamma)) c_{i}}{\left[\gamma \beta\left(1+i-i^{2}\right)+i-\beta\right] C_{1}^{\lambda, \mu}(i)} z^{i}-\sum_{k=m+1}^{\infty} \frac{(1-\beta(1-\gamma))\left(1-\sum_{i=1}^{m} c_{i}\right)}{\left[\gamma \beta\left(1+k-k^{2}\right)+k-\beta\right] C_{1}^{\lambda, \mu}(k)} \sigma_{k} z^{k} \\
& =f_{m}(z)-\sum_{k=m+1}^{\infty}\left(z-\sum_{i=2}^{m} \frac{(1-\beta(1-\gamma)) c_{i}}{\left[\gamma \beta\left(1+i-i^{2}\right)+i-\beta\right] C_{1}^{\lambda, \mu}(i)} z^{i}-f_{k}(z)\right) \sigma_{k} \\
& =f_{m}(z)-\sum_{k=m+1}^{\infty}\left(f_{m}(z)-f_{k}(z)\right) \sigma_{k} \\
= & \left(1-\sum_{k=m+1}^{\infty} \sigma_{k}\right) f_{m}(z)+\sum_{k=m+1}^{\infty} \sigma_{k} f_{k}(z)=\sum_{k=m}^{\infty} \sigma_{k} f_{k}(z) .
\end{aligned}
$$

Corollary 4 The extreme points of the class $A_{\gamma, c_{m}}^{\lambda, \mu, \nu}(1, \beta)$ are the functions $f_{k}(z)(k \geq$ $m$ ) defined by (5.4), (5.5).

Now, we obtain the radii of starlikeness and convexity for the elements of the class $A_{\gamma, c_{m}}^{\lambda, \mu}(1, \beta)$.

Theorem 12 Let the function $f(z)$ defined by (5.1) be in the class $A_{\gamma, c_{m}}^{\lambda, \mu, \nu}(1, \beta)$. Then $f(z)$ is starlike of order $\eta(0 \leq \eta<1)$ in $|z|<\gamma$, where $\gamma$ is the largest value such that

$$
\begin{gathered}
\sum_{i=2}^{m} \frac{c_{i}}{\left[\gamma \beta\left(1+i-i^{2}\right)+i-\beta\right] C_{1}^{\lambda, \mu}(i)} r^{i-1} \\
+\frac{1-\sum_{i=2}^{m} c_{i}}{\left[\gamma \beta\left(1+k-k^{2}\right)+k-\beta\right] C_{1}^{\lambda, \mu}(k)} r^{k-1}<\frac{1}{1-\beta(1-\gamma)} .
\end{gathered}
$$


Proof. It is sufficient to show that

$$
\left|\frac{z f^{\prime}(z)}{f(z)}-1\right|<1-\eta \text {. }
$$

Thus, we have

$$
\begin{gathered}
\left|\frac{z f^{\prime}(z)}{f(z)}-1\right| \leq \\
\frac{\sum_{i=2}^{m} \frac{(i-1)(1-\beta(1-\gamma)) c_{i}}{\left[\gamma \beta\left(1+i-i^{2}\right)+i-\beta\right] C_{1}^{\lambda, \mu}(i)}|z|^{i-1}+\sum_{k=m+1}^{\infty}(k-1) a_{k}|z|^{k-1}}{1-\sum_{i=2}^{m} \frac{\left(1-\beta(1-\gamma) c_{i}\right.}{\left[\gamma \beta\left(1+i-i^{2}\right)+i-\beta\right] C_{1}^{\lambda, \mu}(i)}|z|^{i-1}-\sum_{k=m+1}^{\infty} a_{k}|z|^{k-1}} \\
<\frac{\sum_{i=2}^{m} \frac{(i-1)(1-\beta(1-\gamma)) c_{i}}{\left[\gamma \beta\left(1+i-i^{2}\right)+i-\beta\right] C_{1}^{\lambda, \mu}(i)} r^{i-1}+\sum_{k=m+1}^{\infty} \frac{(k-1)(1-\beta(1-\gamma))\left(1-\sum_{i=2}^{m} c_{i}\right)}{\left[\gamma \beta\left(1+k-k^{2}\right)+k-\beta\right] C_{1}^{\lambda, \mu}(k)} r^{k-1}}{1-\sum_{i=2}^{m} \frac{(1-\beta(1-\gamma)) c_{i}}{\left[\gamma \beta\left(1+i-i^{2}\right)+i-\beta\right] C_{1}^{\lambda, \mu}(i)} r^{i-1}-\sum_{k=m+1}^{\infty} \frac{(1-\beta(1-\gamma))\left(1-\sum_{i=2}^{m} c_{i}\right)}{\left[\gamma \beta\left(1+k-k^{2}\right)+k-\beta\right] C_{1}^{\lambda, \mu}(k)} r^{k-1}} .
\end{gathered}
$$

Therefore (5.6) holds true if the last term of above relationship is less than $1-\eta$ or equivalently

$$
\begin{gathered}
\sum_{i=2}^{m} \frac{(i-\eta)(1-\beta(1-\gamma)) c_{i}}{(1-\eta)\left[\gamma \beta\left(1+i-i^{2}\right)+i-\beta\right] C_{1}^{\lambda, \mu}(i)} r^{i-1} \\
+\sum_{k=m+1}^{\infty} \frac{(k-\eta)(1-\beta(1-\gamma))\left(1-\sum_{i=2}^{m} c_{i}\right)}{(1-\eta)\left[\gamma \beta\left(1+k-k^{2}\right)+k-\beta\right] C_{1}^{\lambda, \mu}(k)} r^{k-1}<1 .
\end{gathered}
$$

Finally we find

$$
\begin{gathered}
\sum_{i=2}^{m} \frac{c_{i}}{\left[\gamma \beta\left(1+i-i^{2}\right)+i-\beta\right] C_{1}^{\lambda, \mu}(k)} r^{i-1} \\
+\frac{1-\sum_{i=2}^{m} c_{i}}{\left[\gamma \beta\left(1+k-k^{2}\right)+k-\beta\right] C_{1}^{\lambda, \mu}(k)} r^{k-1}<\frac{1}{1-\beta(1-\gamma)}
\end{gathered}
$$

and this completes the proof.

Making use of (1.4), we obtain the following corollary.

Corollary 5 Let $f \in A_{\gamma, c_{m}}^{\lambda, \mu, \nu}(1, \beta)$. Then $f$ is convex of order $\eta(0 \leq \eta<1)$ in $|z|<r$ where $r$ is the largest value for which

$$
\begin{gathered}
\sum_{i=2}^{m} \frac{i c_{i}}{\left[\gamma \beta\left(1+i-i^{2}\right)+i-\beta\right] C_{1}^{\lambda, \mu}(i)} r^{i-1} \\
+\frac{k\left(1-\sum_{i=2}^{m} c_{i}\right)}{\left[\gamma \beta\left(1+k-k^{2}\right)+k-\beta\right] C_{1}^{\lambda, \mu}(k)} r^{k-1}<\frac{1}{1-\beta(1-\gamma)} .
\end{gathered}
$$


Theorem 13 Let $f \in A_{\gamma, c_{m}}^{\lambda, \mu, \nu}(1, \beta)$ and

$$
d_{i}=\frac{(1-\beta(1-\gamma)) c_{i}^{2}}{\left|\gamma \beta\left(1+i-i^{2}\right)+i-\beta\right| C_{1}^{\lambda, \mu}(i)}, \quad(2 \leq i \leq m) .
$$

Then the function

$$
h(z)=z-\sum_{i=2}^{m} \frac{(1-\beta(1-\gamma)) d_{i}}{\left(\gamma \beta\left(1+i-i^{2}\right)+i-\beta\right) C_{1}^{\lambda, \mu}(i)} z^{i}-\sum_{k=m+1}^{\infty} a_{k} z^{k}
$$

is also in $A_{\gamma, c_{m}}^{\lambda, \mu, \nu}(1, \beta)$.

Proof. It can be verified that $\left|\gamma \beta\left(1+i-i^{2}\right)+i-\beta\right| C_{1}^{\lambda, \mu}(i)>1, \quad i=2,3, \cdots, m$. Therefore

$$
0 \leq d_{i}=\frac{(1-\beta(1-\gamma)) c_{i}^{2}}{\left|\gamma \beta\left(1+i-i^{2}\right)+i-\beta\right| C_{1}^{\lambda, \mu}(i)}<c_{i} \leq 1 .
$$

So

$$
0 \leq \sum_{i=2}^{m} d_{i}<\sum_{i=2}^{m} c_{i} \leq 1
$$

Thus

$$
\begin{aligned}
& \sum_{k=m+1}^{\infty} \frac{\left[\gamma \beta\left(1+k-k^{2}\right)+k-\beta\right]}{(1-\beta(1-\gamma))\left(1-\sum_{i=2}^{m} d_{i}\right)} C_{1}^{\lambda, \mu}(k) a_{k} \\
< & \sum_{k=m+1}^{\infty} \frac{\left[\gamma \beta\left(1+k-k^{2}\right)+k-\beta\right]}{(1-\beta(1-\gamma))\left(1-\sum_{i=2}^{m} c_{i}\right)} C_{1}^{\lambda, \mu}(k) a_{k}<1
\end{aligned}
$$

and this completes the proof.

\section{References}

[1] E.S. Aqlan, Some Problems Connected with Geometric Function Theory, Ph.D. Thesis, Pune University, Pune, 2004.

[2] W. G. Atshan, Subclass of meromorphic functions with positive coefficients defined by Ruscheweyh derivative II, Surveys in Mathematics and its Applications, vol. 3, 2008, 67-77.

[3] W. G. Atshan, Application of fractional calculus operators for a new class of univalent functions with negative coefficients defined by Hohlov operator, Mathematica Slovaca, vol.60, no.1, 2010, 75-82.

[4] W. G. Atshan, R. H. Buti, Fractional calculus of a class of univalent functions with negative coefficients defined by Hadamard product with Rafid-Operator, European Journal of Pure and Applied Mathematics, vol.4, no.2, 2011, 162173. 
[5] W. G. Atshan, S. R. Kulkarni, Subclass of meromorphic functions with positive coefficients defined by Ruscheweyh derivative I, J. Rajasthan Acad. Phy. Sci., vol.6, no.2, 2007, 129-140.

[6] W. G. Atshan, S. R. Kulkarni, Application of fractional derivatives and hypergeometric functions to multivalent analytic functions, Far East Journal of Mathematical Sciences (FJMS), vol. 27, no. 2, 2007, 277-294.

[7] W. G. Atshan, S. R. Kulkarni, Neighborhoods and partial sums of subclass of $k$ uniformly convex functions and related class of $k$-starlike functions with negative coefficients based on integral operator, Southeast Asian Bulletin of Mathematics, vol. 33, 2009, 623-637.

[8] W. G. Atshan, S. R. Kulkarni, On application of differential subordination for certain subclass of meromorphically p-valent functions with positive coefficients defined by linear operator, Journal of Inequalities in Pure and Applied Mathematics, vol. 10, no. 2, 2009, Article 53, 11 pp.

[9] P. L. Duren, Univalent Functions, Grundelheren der Mathematischen Wissenchaften 259, Springer - Verlag, New York, Berlin, Heidelberg, Tokyo, 1983.

[10] S. Kanas, A. Wisniowska, Conic regions and k-uniformly convexity II, Folia Sci. Tech. Reso., 178, 1998, 65-78.

[11] Y. Komato, On analytic prolongation of a family of operators, Mathematica (Cluj), vol. 39, no. 55, 1990, 141-145.

[12] M. Nunokawa, A sufficient condition for univalence and starlikeness, Proc. Japan Acad. Ser. A Math. Sci., vol. 65, 1989, 163-164.

[13] S. Owa, M. Nunokawa, H. M.Srivastava, A certain class of multivalent functions, Appl. Math. Lett., vol. 10, no. 2, 1997, 7-10.

[14] T. Rosy, K. G. Subramanian, G. Murugusundaramoorthy, Neighbourhoods and partial sums of starlike functions based on Ruscheweyh derivatives, J. Inequal. Pure and Appl. Math., vol. 4, no. 4, 2003, 1-8.

[15] S. Shams, S. R. Kulkarni, A class of univalent functions with negative and fixed finitely many coefficients, Acta Cienica Indica, XXXIXM, vol. 3, 2003, 587-594.

[16] H. Silverman, Univalent functions with negative coefficients, Proc. Amer. Math. Soc., vol. 51, 1975, 109-116.

[17] H. M. Srivastava, Distortion Inequalities for analytic and univalent functions associated with certain fractional calculus and other linear operators, (In Analytic and Geometric Inequalities and Applications eds. T. M. Rassias and H. M. Srivastava), Kluwar Academic Publishers, vol. 478, 1999, 349-374. 
[18] H. M. Srivastava, R. K. Saxena,Operators of fractional integration and their applications, Applied Mathematics and Computation, vol. 118, 2001, 1-52.

[19] A. Tehranchi, S. R. Kulkarni, Study of the class of univalent functions with negative coefficients defined by Ruscheweyh derivatives (II), J. Rajasthan Academy of Physical Sciences, vol. 5, no. 1, 2006, 105-118.

\section{Waggas Galib Atshan}

University of Al-Qadisiyah

College of Science

Department of Mathematics

Diwaniya, Iraq

e-mail:waggashnd@gmail.com, waggas.galib@qu.edu.iq

\section{S. R. Kulkarni}

University of Pune

Fergusson College

Department of Mathematics

Pune - 411004, India

e-mail:kulkarni_ferg@yahoo.com 